\title{
Biomechanical Conceptual Design of a Passive Transfemoral Prosthesis
}

\author{
R. Unal, R. Carloni, E.E.G. Hekman, S. Stramigioli and H.F.J.M. Koopman
}

\begin{abstract}
In this study, we present the conceptual design of a fully-passive transfemoral prosthesis. The proposed design is inspired by the analysis of the musculo-skeletal activity of the healthy human leg. In order to realize an energy efficient device, we introduce three storage elements, which are responsible of the energetic coupling between the knee and the ankle joints. Simulation results show that the power storage of the designed conceptual prosthesis is comparable with the human gait.
\end{abstract}

\section{INTRODUCTION}

The main research challenges in the design of transfemoral prostheses are the efficiency with respect to the metabolic/external energy consumption and the adaptability to various walking conditions. In both literature and market, different kinds of transfemoral prostheses are present and they can be classified as follows:

- passive, i.e. not actuated - These prostheses can be considered efficient from the mechanical point of view but the overall efficiency is hampered by the considerable amount of extra metabolic energy consumption [1]. Moreover, due to the constant mechanical characteristics, these devices can not adapt to different conditions.

- controlled by means of internal, intrinsically passive, actuators - These prostheses use external power to adapt their dynamics to different gait pattern. For example, in [2] and [3], the dynamical behavior of the prosthesis during walking relies on the control of a magnetorheological damper, which produces the required breaking torque for the knee joint.

- active (powered), i.e. actuated - These prostheses are capable to inject power in order to provide active ankle push-off generation, so to reduce the extra metabolic energy consumption [4], [5], [6], [7].

Recently, some of the design studies have been focused on the transfemoral prosthesis with energy storage capabilities in order to reduce the power consumption. For example, in [8], [9] and [10], energy storage and release are provided by using an adjustable spring. Electrically powered transfemoral prostheses include a spring in parallel to the ankle motor unit and initial tests have been reported in [11]. Additionally, the design studies on soft actuators have shown that the energy efficiency of the system can be improved by storing the energy during stance phase and by releasing it so to provide active ankle push-off generation [12], [13].

This work has been partially funded by the Dutch Technology Foundation STW as part of the project REFLEX-LEG under the grant no. 08003.

\{r.unal,r.carloni,s.stramigioli\}@utwente.nl, Faculty of Electrical Engineering, Mathematics and Computer Science, University of Twente, The Netherlands.

\{r.unal,e.e.g.hekman,h.f.j.m.koopman\}@utwente.nl, MIRA Institute, Faculty of Engineering Technology, University of Twente, The Netherlands.
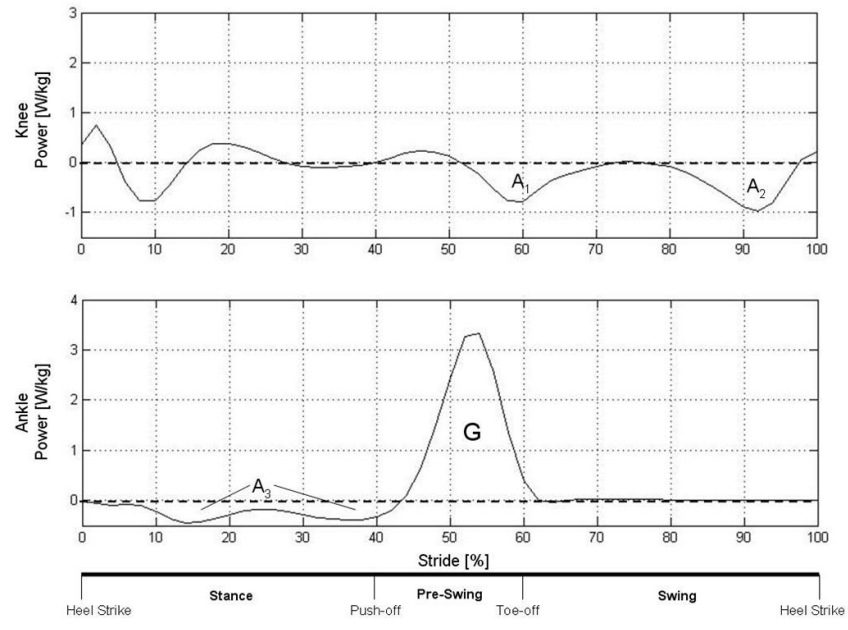

Fig. 1. The power flow of the healthy human gait normalized in body weight in the knee (upper) and the ankle (lower) joints during one stride [15] The areas $A_{1,2,3}$ indicate the energy absorption, whereas $G$ indicates the energy generation. The cycle is divided into three phases (stance, pre-swing and swing) with three main instants (heel-strike, push-off and toe-off).

In this paper, we propose a biomechanical conceptual design of a fully-passive energy efficient transfemoral prosthesis. The concept is mainly based on mimicking the energetic behavior of a human gait in terms of coupling the energy absorption and generation of the knee and ankle joints by means of three energy storage elements. This study has been introduced in our previous work [14], and here we intend to improve the working principle so to obtain a comparable power storage capability between the human leg and the proposed prosthetic device. Promising results that promotes the conceptual design, have been obtained by simulation.

\section{ANALYSIS OF THE HUMAN GAIT}

In order to grasp the nature of walking, we analyze the biomechanical data of the human gait, as been presented by Winter in [15]. In particular, Fig. 1 depicts the power flow at the knee (upper) and ankle (lower) joints during one complete stride of a healthy human, normalized in body weight. The figure highlights three instants, i.e. heel strike, push-off and toe-off, and three main phases:

- Stance: the knee absorbs a certain amount of energy during flexion and generates as much as the same amount of energy for its extension. In the meantime, the ankle joint absorbs energy, represented by $A_{3}$ in the figure, due to the weight bearing.

- Pre-swing: the knee starts absorbing energy, represented by $A_{1}$ in the figure, while the ankle generates the main 
part of the energy for the push-off, represented by $G$, which is about the $80 \%$ of the overall generation.

- Swing: the knee absorbs energy, represented by $A_{2}$ in the figure, during the late swing phase, while the energy rate in the ankle joint is negligible.

Note that, in the healthy human gait, the knee joint is mainly an energy absorber whereas the ankle joint is mainly an energy generator. Moreover, there is almost a complete balance between the generated and the absorbed energy, since the energy for push-off generation, i.e. $G$, is almost the same as the total energy absorbed in the three intervals $A_{1,2,3}$.

This means that, in order to design an energy efficient transfemoral prosthesis, instead of providing all the energy required for ankle push-off from the external actuators or instead of dissipating the energy by using breaks, the design should be such that the ankle directly exploits the energy absorbed by both the knee and ankle joints during the gait. Therefore, we can state that the efficiency of the mechanism derives from an energetic coupling, i.e. an energetic transfer, between the knee and ankle joints.

\section{CONCEPTUAL DESIGN OF THE PROSTHESIS}

In the proposed concept, we introduce three storage elements, which are responsible for the three absorption intervals $A_{1,2,3}$ and the transfer of the energies $A_{1}$ and $A_{2}$ from the knee to the ankle joint. As summarized in Fig. 2, our design relies on:

- One torsional elastic element $\mathbb{C}_{1}$ at the knee joint, responsible for the absorption $A_{1}$ and for its transfer (during swing phase) to the elastic element $\mathbb{C}_{3}$.

- One linear elastic element $\mathbb{C}_{2}$, which physically connects the upper leg, via a lever arm, and the foot. Therefore, it couples the knee and ankle joints. This element is responsible for the absorption $A_{2}$ during the swing phase and for a part of the absorption $A_{3}$ during stance phase.

- One linear elastic element $\mathbb{C}_{3}$, which physically connects the lower leg and the foot and is responsible for the absorption $A_{1}$ (received from $\mathbb{C}_{1}$ ) and a part of $A_{3}$ during stance phase.

It is assumed that the knee joint absorbs and generates the same amount of energy during stance phase, therefore for this phase, the knee joint is not considered as a contributor to the ankle push-off generation. For this reason, an elastic element to mimic this behavior is not included in the design.

\section{A. Energy storage during swing phase}

The swing phase of the human gait is an energy absorption phase for the knee joint and, therefore, the energy absorbed at the knee joint has to be transferred to the ankle joint. For the storage purpose in the swing phase, all the three elastic elements are employed, and their working principle are depicted in Fig. 3 and Fig. 4.

Due to ankle push-off, the lower leg has an amount of kinetic energy equal to $A_{1}$, which is stored in the torsional spring $\mathbb{C}_{1}$ during the backward swing of the lower leg. Once the knee joint reaches full-flexion, the element $\mathbb{C}_{1}$ is locked

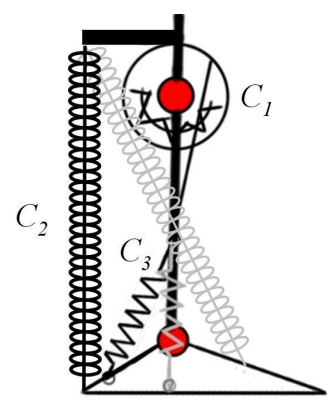

Fig. 2. Conceptual design of the proposed mechanism - The design consists of three storage elements, the torsional spring $\mathbb{C}_{1}$ at the knee joint, the linear spring $\mathbb{C}_{2}$ between the upper leg and foot (via a lever arm) and the linear spring $\mathbb{C}_{3}$ between the lower leg and the foot. Both $\mathbb{C}_{2}$ and $\mathbb{C}_{3}$ are subjected to configuration change.

and, therefore, disengaged from the knee joint without energy dissipation, since the knee joint has zero velocity at this instant. Simultaneously, the elastic element $\mathbb{C}_{3}$ is changing the attachment point from $P_{4}$ to $P_{5}$, after it is unloaded for the push-off (see Fig. 3a and 3b). After that, during the swing phase, the state of the energy storage elements changes as follows:

- The attachment point of the spring $\mathbb{C}_{2}$, which is unloaded, is changed from $P_{1}$ (on the heel) to $P_{2}$ (on the upper foot) in order to store the energy $A_{2}$. At the beginning of the swing motion of the lower leg, the element $\mathbb{C}_{2}$ also provides the necessary ankle dorsiflexion so to guarantee the ground clearance (see Fig. 4 - left).

- Once the ankle joint is fixed for the ground clearance, the element $\mathbb{C}_{1}$ releases the energy $A_{1}$ to the element $\mathbb{C}_{3}$ by changing the attachment point $P_{6}$ of $\mathbb{C}_{3}$ upward along the lower leg (see Fig. 3c). This energy transfer is realized via pulley by aligning the arm for zero torque around the knee joint during swing phase. Therefore, this transfer will not interfere the natural swing motion. Since the design detail of the mechanism is out of scope in this work, Fig. 3 is representing just an illustration of the concept.

At the end of the swing phase, the attachment points of the elements $\mathbb{C}_{2}$ and $\mathbb{C}_{3}$ are changed back to their initial configuration at the heel, i.e. the attachment point of $\mathbb{C}_{2}$ moves back from $P_{2}$ to $P_{1}$ (see Fig. 4 - right) and the attachment point of $\mathbb{C}_{3}$ moves back from $P_{5}$ to $P_{4}$ (see Fig. 3d). These changes guarantee that the total energy $A_{1}$ and $A_{2}$ is stored in the elements $\mathbb{C}_{2}$ and $\mathbb{C}_{3}$ and, therefore, it has been transferred to the ankle joint so to provide support to the ankle push-off generation. Note that to have an energy efficient transfer, the change of the configuration of the elements $\mathbb{C}_{2}$ and $\mathbb{C}_{3}$ should be realized ideally without any dissipation. Therefore, at the heel strike, the attachment points are changed along proper defined trajectories, which keep the length of $\mathbb{C}_{2}$ and $\mathbb{C}_{3}$ constant (without elongation or compression). 

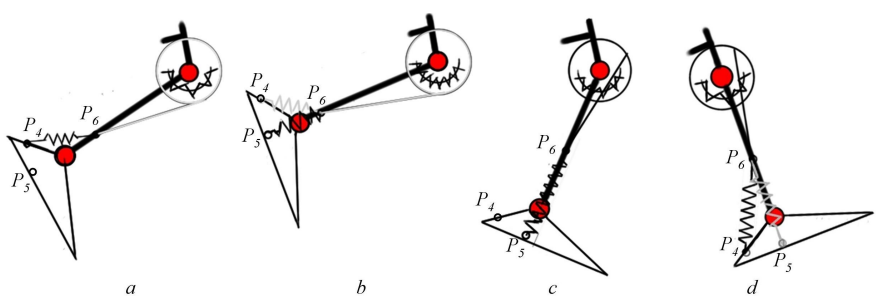

Fig. 3. Configuration change of the storage element $\mathbb{C}_{3}$ during swing phase - (a) After push-off, the energy $A_{1}$ is stored by loading the element $\mathbb{C}_{1}$ on the knee joint. (b) At the full-flexion of knee joint, the energy storage is completed and $\mathbb{C}_{1}$ is locked and disengaged from the knee joint. At this instant, the attachment point of the unloaded element $\mathbb{C}_{3}$ is changed from $P_{4}$ to the $P_{5}$. (c) During swing motion the energy is transferred from $\mathbb{C}_{1}$ to $\mathbb{C}_{3}$ by changing upwards the position of the attachment point of $\mathbb{C}_{3}$ along the lower leg $\left(P_{6}\right)$. (d) After the transfer has been completed, the position of $P_{6}$ is fixed and the element $\mathbb{C}_{3}$ is brought back to stance configuration with the heel-strike (change from $P_{5}$ to $P_{4}$ ). Note that the configuration changes of element $\mathbb{C}_{3}$ take place over a predefined trajectory which keeps the length of the element constant.
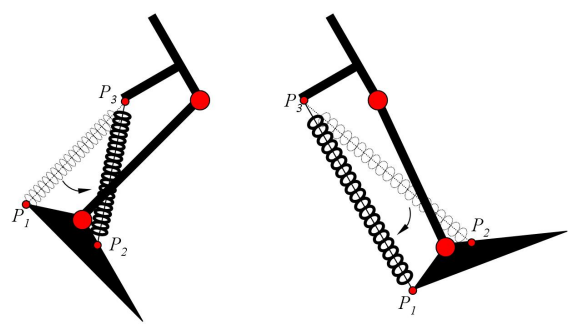

Fig. 4. Configuration change of the storage element $\mathbb{C}_{2}$ during swing phase - After pre-swing phase, the attachment point of the spring $\mathbb{C}_{2}$ is changed from the heel $\left(P_{1}\right)$ to the upper part of the foot $\left(P_{2}\right)$ (left). At the end of the swing, the spring is loaded and its position changes back to the $P_{1}$ (right). The point $P_{3}$ is the attachment point of the spring on the lever arm of the upper leg. Note that the configuration changes of element $\mathbb{C}_{2}$ take place over a predefined trajectory which keeps the length of the element constant.

\section{B. Energy storage during stance phase}

During the stance phase, the state of the energy storage elements $\mathbb{C}_{2}$ and $\mathbb{C}_{3}$ changes as follows:

- The element $\mathbb{C}_{2}$, which is already loaded with the energy $A_{2}$, elongates and absorbs part of the energy $A_{3}$.

- While the ankle joint is in dorsi-flexion motion, a braking torque is applied to the ankle in order to bear the weight of the body. Instead of dissipating the energy by using a brake system, the storage element $\mathbb{C}_{3}$ provides the brake torque and, therefore, stores the corresponding energy $A_{3}$.

This working principle during the stance phase is depicted in Fig. 5.

At the end of the stance phase, the storage elements $\mathbb{C}_{2}$ and $\mathbb{C}_{3}$ are loaded and, therefore, are ready to release the total energy of all absorption phases $\left(A_{1}, A_{2}, A_{3}\right)$ for the ankle push-off. Note that, the first swing storage part $\mathbb{C}_{1}$ is only active during the swing phase. Therefore, there is no undesirable interference of the storage parts during walking.

\section{DESIGN PARAMETERS}

In this Section, we identify the storage element values by using the biomechanical data for a human of $1.8 \mathrm{~m}$ height and $80 \mathrm{~kg}$ weight [16].

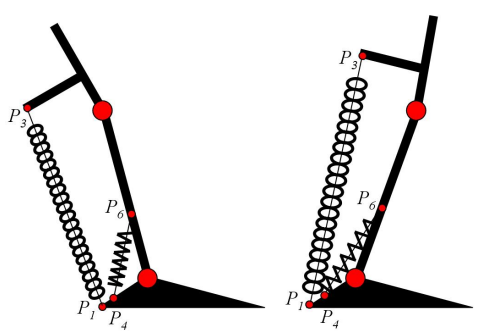

Fig. 5. The working principle at stance phase - At the beginning of the stance phase, both elements $\mathbb{C}_{2}$ and $\mathbb{C}_{3}$ are ready for the storage of the energy $A_{3}$ (left). At the end of the stance phase, both springs are loaded (right).

The elastic constants of the springs employed for the swing phase are derived from the energy values of the absorption intervals $A_{1}$ and $A_{2}$. In particular, the elastic constant $k_{1}$ of the torsional spring $\mathbb{C}_{1}$ is determined from the absorption interval $A_{1}$, i.e.:

$$
A_{1}=\frac{1}{2} k_{1} \delta s_{1}^{2}
$$

where $\delta s_{1}$ is the radial deflection of the torsional spring $\mathbb{C}_{1}$ and is equal to the variation of the knee angle, which is about $0.84 \mathrm{rad}$ during this interval (between $52 \%$ and $72 \%$ of the stride). It follows that $k_{1}=20.88 \mathrm{Nm} / \mathrm{rad}$.

The elastic constant $k_{2}$ of the linear spring $\mathbb{C}_{2}$ is determined from the absorption interval $A_{2}$, i.e.:

$$
A_{2}=\frac{1}{2} k_{2} \delta s_{2}^{2}
$$

where $\delta_{s_{2}}$ is the deflection of the spring $\mathbb{C}_{2}$ and is given by

$$
\delta s_{2}=\left|\mathbf{P}^{\mathbf{P}_{3} \mathbf{P}_{2}}\right|-s_{2_{0}}
$$

where the magnitude of $\mathbf{P}^{\mathbf{P}_{3} \mathbf{P}_{2}}$ is the length of the $\mathbb{C}_{2}$ element when it is attached between $P_{3}$ and $P_{2}$ (see Fig. 4) and $s_{2_{0}}$ is its initial length, which is $0.43 \mathrm{~m}$ at the beginning of swing (see Fig. 4 - left). It follows that $k_{2}=1925.6 \mathrm{~N} / \mathrm{m}$.

During stance phase, the energy is stored in both $\mathbb{C}_{2}$ and $\mathbb{C}_{3}$. It should be noted that, this parallel structure leads to smaller elastic constant for the element $\mathbb{C}_{3}$. During the stance phase, the deflection $\delta s_{2}$ of the storage element $\mathbb{C}_{2}$ is given by

$$
\delta s_{2}=\left|\mathbf{P}^{\mathbf{P}_{3} \mathbf{P}_{1}}\right|-s_{2_{0}}
$$

in which the magnitude of $\mathbf{P}^{\mathbf{P}_{3} \mathbf{P}_{\mathbf{1}}}$ is the length of the element $\mathbb{C}_{2}$ when it is attached between $P_{3}$ and $P_{1}$ (see Fig. 4) and $s_{2_{0}}$ is its initial length, which is $0.52 \mathrm{~m}$ at the end of swing (see Fig. 4 - right). The deflection $\delta s_{3}$ of the stance storage element is given by

$$
\delta_{s_{3}}=\left|\mathbf{P}^{\mathbf{P}_{6} \mathbf{P}_{\mathbf{4}}}\right|-s_{3_{0}}
$$

in which the magnitude of $\mathbf{P}^{\mathbf{P}_{6}} \mathbf{P}_{\mathbf{4}}$ is the length of the element $\mathbb{C}_{3}$, attached between $P_{6}$ and $P_{4}$ (see Fig. 5), and $s_{3_{0}}$ is its initial length, which is $0.16 \mathrm{~m}$ at the beginning of rollover (see Fig. 5 - left). The elastic constant $k_{3}$ of the stance storage element $\mathbb{C}_{3}$ can be found from the energy value of the absorption interval $A_{3}$, i.e.

$$
A_{3}=\frac{1}{2} k_{2} \delta s_{2}{ }^{2}+\frac{1}{2} k_{3} \delta s_{3}{ }^{2}
$$


where $k_{2}$ is the elastic constant of the storage element $\mathbb{C}_{2}$. It follows that $k_{3}=82500 \mathrm{~N} / \mathrm{m}$.

\section{SIMULATION AND RESULTS}

In this Section, we simulate the conceptual prosthesis in Matlab/Simulink environment. The dynamic model has been derived by using Kane's method [17]. To demonstrate the power absorption performance of the mechanism, the simulation has been done for the swing and stance phases separately. Note that, since the model has been built to see the feasibility of the conceptual design, all the mechanical losses and mass of the elastic elements are neglected.

For the simulation of the swing phase, we apply the hip torque of a healthy human [15] to the device as an external input, while for the simulation of the stance phase, in addition to the hip torque, we apply the forces of the sound leg [15] to the torso as an external input.

Fig. 6 illustrates the power storage profile of the conceptual mechanism (continuous lines) compared to the healthy human gait (dashed line) [15]. The figure shows that the profile of the power storage of the mechanism matches quite well with the healthy human gait. The power analysis shows that the $66 \%$ of the absorption interval $A_{1}$, the $70 \%$ of the absorption interval $A_{2}$ and the $88 \%$ of the absorption interval $A_{3}$ can be stored with the proposed conceptual mechanism during natural gait. Therefore, overall the $76 \%$ of the possible amount of energy can be harvested from walking in order to realize ankle push-off generation. On top of this energy, extra energy should be injected to the system in order to realize the ankle push-off generation. Since the system is fully passive, this energy will be generated with extra torque from the hip and the extra forces from the sound leg. The application of the forces and torques to compensate this energy is dependent on the human adaptation. Even though there will be mechanical losses in the mechanism, the total amount of energy that can be stored in the system indicates significant support for the push-off, which will reduce the metabolic energy consumption of the amputee.

\section{CONCLUSIONS AND FUTURE WORKS}

In this study, we proposed a biomechanical conceptual mechanism, inspired by the power flow in the human gait, for a transfemoral prosthesis. The conceptual mechanism is build up with three elastic storage elements, which properly create a coupling between the knee and ankle joints so to obtain an efficient energy transfer between the joints. The working principle has been evaluated in simulation and the results show that the power storage capability of the mechanism is comparable with the healthy gait. Therefore, this study shows the feasibility of the concept towards the realization of an energy efficient transfemoral prosthesis.

Future work will focus on the design of the concept, which will be improved by optimizing the design parameters according the maximum power storage capabilities. Minimum mechanical losses and control of the release rate of the elastic elements will also be taken into account during the realization of the prosthetic device.
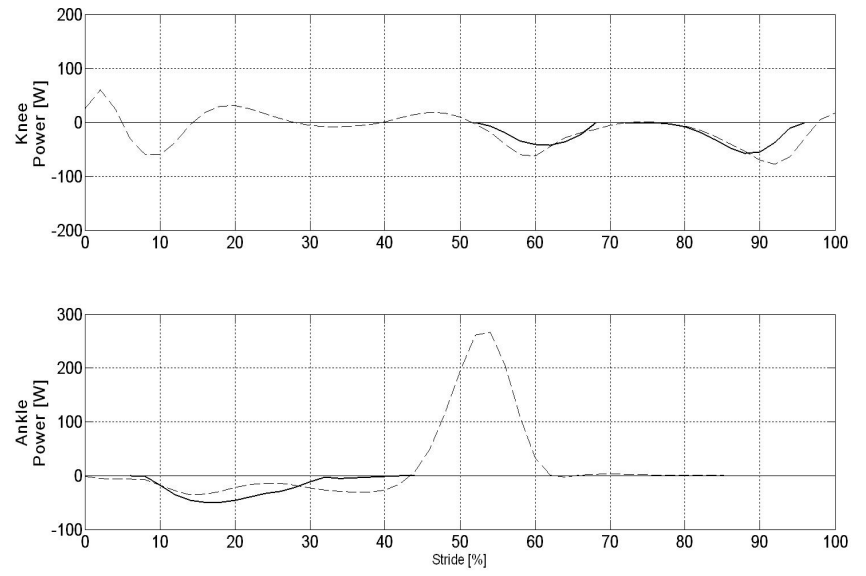

Fig. 6. The power flow of the healthy human gait [15] (dashed line) and the power flow for the conceptual mechanism (continuous lines for the three storage elements) during one stride (for a human of $1.8 \mathrm{~m}$ height and $80 \mathrm{~kg}$ weight [16])

\section{REFERENCES}

[1] R. Waters, J. Perry, D. Antonelli and H. Hislop, "Energy Cost of Walking Amputees: The Influence of Level of Amputation", Jour. Bone and Joint Surgery, vol. 58A, pp. 42-46, 1976.

[2] J.H. Kim and J.H. Oh, "Development of an Above Knee Prosthesis Using MR Damper and Leg Simulator", IEEE Int. Conf. on Robotics and Automation, 2001.

[3] H. Herr and A. Wilkenfeld, "User-adaptive Control of a Magneto Rheological Prosthetic Knee", Industrial Robot: An Int. Jour., vol. 30, pp. 42-55, 2003.

[4] F. Sup, A. Bohara and M. Goldfarb, 'Design and Control of a Powered Transfemoral Prosthesis", Int. Jour. Robotics Research, vol. 27, pp. 263-273, 2008

[5] W.C. Flowers, "A Man-Interactive Simulator System for Above-Knee Prosthetics Studies", PhD Thesis, MIT, 1973.

[6] D. Popovic and L. Schwirtlich, "Belgrade Active A/K Prosthesis", in de Vries, J. (Ed.), Electrophysiological Kinesiology, Int. Congress, Excerpta Medica, pp. 337-343, 1988.

[7] S. Bedard and P. Roy, "Actuated Leg Prosthesis for Above-Knee Amputees", 7314490 US Patent, 2003.

[8] A. Rovetta, M. Canina, P. Allara, G. Campa and S.D. Santina, "Biorobotic design criteria for innovative limb prosthesis", Int. Conf. on Advanced Robotics, 2001.

[9] A. Rovetta, T. Chettibi and M. Canina, "Development of a Simple and Efficient Above Knee Prosthesis", IMECE Int. Sym. Advances in Robot Dynamics and Control, 2003.

[10] M. Canina and A. Rovetta, 'Innovatory Bio-robotic System for the Accumulation of the Energy of Step in a Limb prosthesis", Int. Workshop Robotics in Alpe-Adria-Danube Region, 2003.

[11] F. Sup, H.A. Varol, J. Mitchell, T. Withrow and M. Goldfarb, "SelfContained Powered Knee and Ankle Prosthesis: Initial Evaluation on a Transfemoral Amputee", IEEE Int. Conf. on Rehabilitation Robotics, 2009.

[12] K. W. Hollander, T.G. Sugar and D. E. Herring, "Adjustable robotic tendon using a 'Jack Spring'TM", IEEE Int. Conf. on Rehabilitation Robotics, 2005.

[13] R. Bellman, A. Holgate and T.G. Sugar, "SPARKy 3: Design of an Active Robotic Ankle Prosthesis with Two Actuated Degrees of Freedom Using Regenerative Kinetics", IEEE/RAS-EMBS Int. Conf. on Biomedical Robotics and Biomechatronics, 2008.

[14] R. Unal, R. Carloni, E.E.G. Hekman, S. Stramigioli and H.F.J.M. Koopman, "Conceptual Design of an Energy Efficient Transfemoral Prosthesis", IEEE/RSJ International Conference on Intelligent Robots and Systems, 2010.

[15] D.A. Winter, The Biomechanics and Motor Control of Human Gait: Normal, Elderly, and Pathological, University of Waterloo Press, 1991.

[16] J. Rose and J.G. Gamble, Human Walking, Williams \& Wilkins, 2005

[17] T.R. Kane, Dynamics, Theory and Applications, McGraw-Hill, 1985. 